\title{
A Study of Water Safety Plan (WSP) For Environmental Risk Management of a Modern North Indian City
}

\author{
Harish Kumar Saini1 ${ }^{1,}$ Dr. R.K. Khitoliya ${ }^{2}$ Dr. Shakti Kumar ${ }^{3,}$ \\ ${ }^{I}$ (M.E. $2^{\text {nd }}$ year Student, Civil Engineering Department, PEC University of Technology, Chandigarh, India) \\ 2 (Professor and Head, Civil Engineering Department, PEC University of Technology, Chandigarh, India) \\ ${ }^{3}$ (Associate Professor, Civil Engineering Department, PEC University of Technology, Chandigarh, India)
}

\begin{abstract}
Access to safe drinking water is a basic need for human development, health and well being and because of this it is an internationally accepted human right (WHO, 2011).

There has been encouraging progress with access to safe drinking water \& sanitation in both rural and urban areas since the United Nations water decade of the 1980s. However, more than 1 billion people around the world still lack access to safe water supplies. A substantial majority of these people live in Asia.

Water has many constituents. Some of these are of natural origin \& some are manmade and present as contaminants. Some give rise to serious health effects and some will give rise to only aesthetic characteristics.

Over the past decade it has become increasingly evident that the delivery of safe \& aesthetically acceptable drinking water cannot be assured if it is based on only measurements of water quality parameters \& or the performance of a water filtration plant.

In view of the uncertainties and the practical difficulty in monitoring the quality of treated water, a risk based approach to managing the quality of drinking water is required. A key advantage of a risk based approach is in avoiding the costs associated with installing inappropriate systems of delivering water. It has been shown that investments in water supply and sanitation can yield a net economic benefit, as the reductions in adverse health effects and health care costs outweigh the costs of undertaking these interventions.

The guidelines provided in this thesis are an important contribution to environmental risk assessment and the avoidance of the physical, chemical and microbial contamination of drinking water.

The HACCP plan from which this water safety plan extract has been derived is scoped to cover the entire water system from catchment to tap and is dynamic document continually evolving as increased knowledge and experience and present opportunities for improvement.

Water safety management demands a quantitative understanding of how processes \& actions effect water quality, which in turn requires an understanding of environmental risk assessment. This study is intended to provide guidance on using environmental risk assessment while developing water safety plan to ensure the production of high quality drinking water in a modern North Indian City like Chandigarh.
\end{abstract}

\section{Introduction}

Managing water resources and supplying safe water are the greatest challenges for the present generation. What makes water safe is the care and consideration people have for activities and actions in the catchment and in treatment, storage and distribution of water.

Water suppliers and Municipalities have a public health responsibility to these communities to provide drinking water that is safe to drink. Safe drinking water is a basic need for human development, health \& wealth being and because of this is an internationally accepted human right (WHO, 2001).

The most effective means of consistency exercising the safety of a drinking water supply is through the use of comprehensive risk assessment $\&$ risk management approach that includes all steps in water supply from catchment to consumers, such approaches are called WATER SAFETY PLANS (WSP).

It is based on the principals of Hazard Analysis and critical control points (HACCP) widely used in Food Industry. In the Food Industry, a HACCP Plan seeks to protect food quality from FARM TO THE FORK. In the water industry, a WSP is a comprehensive approach from CATCHMENT TO CONSUMER.

The WHO has developed guidelines for drinking water quality. The latest edition of the WHO Guidelines for drinking water quality is structured around on overall "Water Safety Framework" used to develop supply specific "Water Safety Plans".

Preparing a water safety plans involves a systematic assessment of every aspect of providing safe drinking water, identifying the events that could cause water to become unsafe to drink \& developing plans to manage these.

The present research provides processes for identifying potential problems and managing risks to water quality in a developed modern north Indian city like Chandigarh. 


\section{Key Components of WSP}

\section{Methodology}

There are 11 modules involved in the developing \& implementation of Water Safety Plan (WSP).

Preliminary and preparation

System Risk Assessment

Module-1 (Assemble WSP Team)

Module-3 (Hazard identification \& initial risk assessment)

Module-4 (Control measures and reassess risk)

System Upgrade

Quality monitoring \& verification

Module-5 (Improvement Plan)

Module-6 (Control measure monitoring)

Module-7 (Verification)

Supporting WSP Management

$\&$ verification Procedures

Module-8 (Management procedure)

Feedback/ Reviews

Module-9 (Supporting programmes)

Module-11 (Incidents)

Module-10 (Review)

Based on the above Modules, the Water Safety Plan has been developed for Chandigarh city, which is a modern city of North India known as "THE CITY BEAUTIFUL".

\section{Description of Water Supply System in Chandigarh}

As per Module-2, it is necessary to understand and describe the water supply system of Chandigarh right from catchment, pumping, treatment, distribution and upto the consumer end (Household).

There are two sources of raw water supply in city Chandigarh:

1. 80 MGD surface water pumped from Bhakra Canal at a distance of $27 \mathrm{KM}$ from Chandigarh.

2. 20 MGD ground water from deep bore tubewells located across the city.

The raw water is treated at water works sector 39 (known as Mother Water Works) \& water works Sector 12 is pumped to all other Water Works located in Sector 12, 26, 32, $37 \& 52$ which act as intermediate pumping stations for supplying the treated water to their localized areas/ zones as illustrated in the following flow diagram:

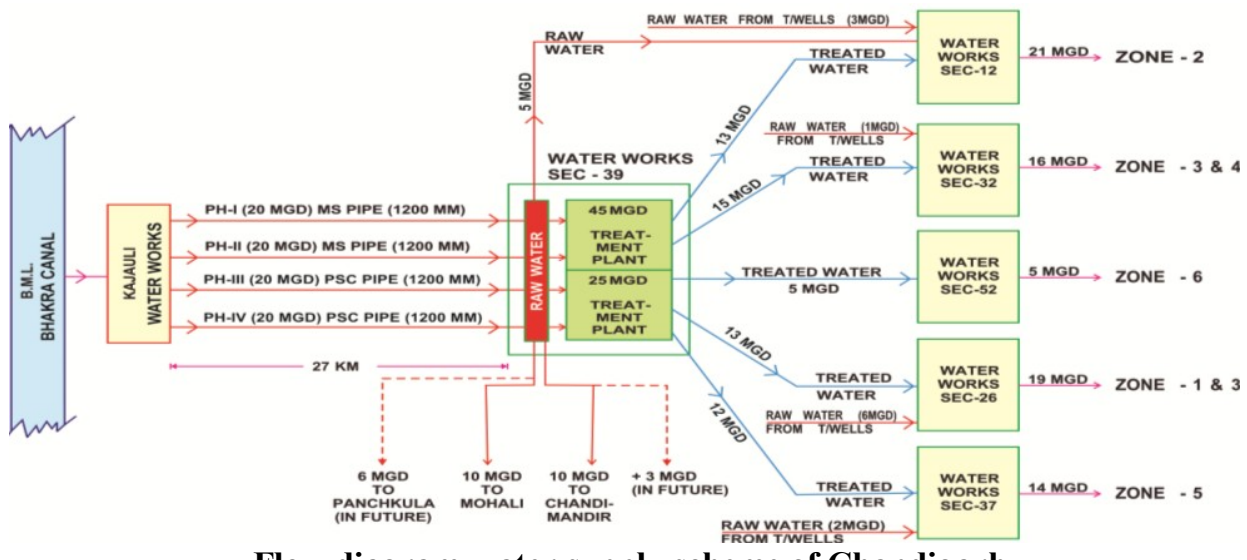

Flow diagram-water supply scheme of Chandigarh

\section{Selection of Pilot Zone (Study area)}

Out of 6 zones in which Chandigarh is divided for its water supply distribution, Zone No 2 has been selected as Pilot Zone for initial development \& implementation of Water Safety Plan in city Chandigarh. Zone No 2 is being fed through Water Works Sector 12 \& supply drinking water to Sector 12 to $18,21-\mathrm{A}, 22$ A \& B, 25.

3.1 Intake of Water in WTP of Pilot Zone No 2

i. 5 MGD of raw water from Bhakra Canal through the Water Works Sector 39.

ii. 3 MGD of raw water from 19 No deep bore tubewells (Ground water).

iii. 13 MGD of treated water from Water Works Sector 39.

5 MGD raw water received at Water Works Sector 12 is being treated in different stages (Coagulation, Flocculation, Sedimentation, Filtration and Post Chlorination).

Water supply treatment process/ distribution in Pilot Zone 2 as illustrated in the following flow diagram: 


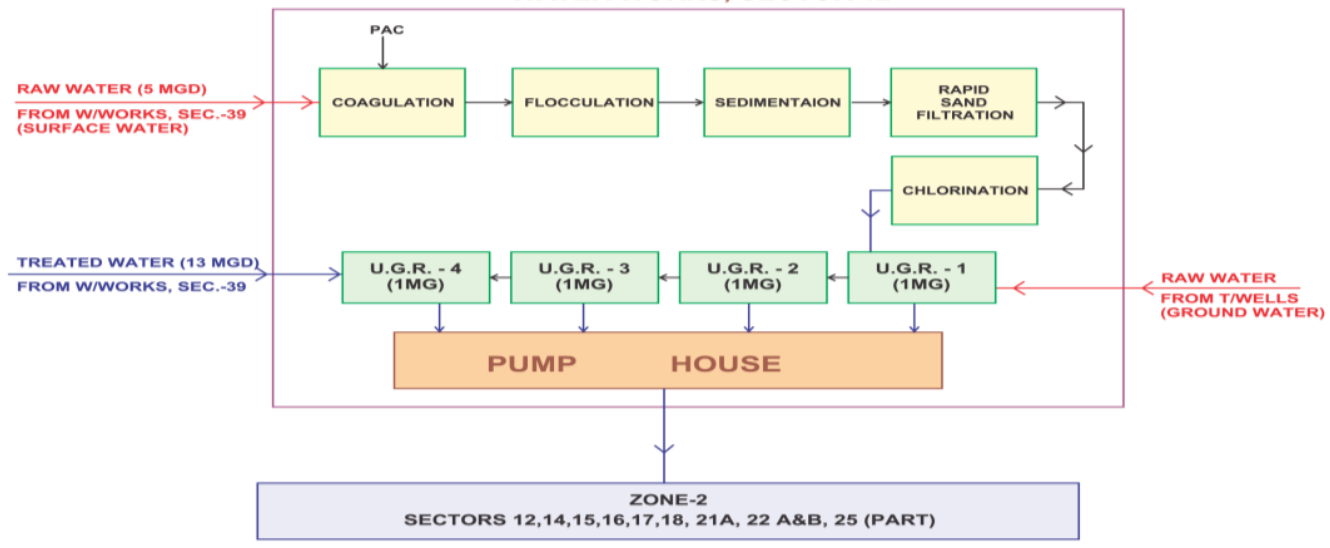

Flow diagram water supply distribution in pilot zone 2 (From water works sector 12)

4. Summary of Historical Water Quality Data for Pilot Zone

The data for the five years (2008 to 2012) has been analyzed for the samples taken from:

i. Raw Water.

ii. Treated water (At outlet of WTP i.e. Water Works Sector 12).

iii. Distribution system-cum-consumer end.

For each year; average, maximum, minimum, standard variation and median values have been calculated for $\mathrm{pH}$, Turbidity, Hardness, Total dissolved solids \& for free Residual Chlorine available.

\section{Results And Discussion}

\section{Analysis of treated water data at Water Works Sector 12 (Pilot Zone)}

For the year 2013-14, the daily sampling data for treated water at the outlet of WTP Sector 12 has been analyzed and the variation in maximum recorded values and its desirable limits as per IS: 10500:2012 are as under:

Variations (Actual data Vs acceptable limits) For Treated Water at outlet of Water Works Sector-12

\begin{tabular}{|l|l|c|c|c|}
\hline Year & \multicolumn{1}{|c|}{ Parameter } & $\begin{array}{c}\text { Maximum } \\
\text { recorded data }\end{array}$ & $\begin{array}{c}\text { Desirable limit as per IS } \\
\text { 10500: 2012 }\end{array}$ & Remarks \\
\hline 2014 & $\mathrm{pH}$ & 7.87 & $6.5-8.5$ & $\begin{array}{c}\text { Within } \\
\text { limits }\end{array}$ \\
\hline & Turbidity (NTU) & 1.20 & 5 & -do- \\
\hline & TDS (mg 1$)$ & 190 & 500 & -do- \\
\hline & Hardness (mg 1$)$ & 110 & 300 & -do- \\
\hline & Chlorides (mg 1$)$ & 6.0 & 250 & -do- \\
\hline & FRC (mg 1$)$ & 0.3 & 0.2 & -do- \\
\hline & $\begin{array}{l}\text { MPN Coliform } \\
\text { Index }\end{array}$ & 0 & 0 & -do- \\
\hline
\end{tabular}

2. Analysis of water quality historical data in pilot zone

Historical sampling data for the 5 years (2008-2012) has been analyzed for raw water, treated water (at outlet of WTP Sector 12) \& distribution system-cum-consumer end \& the results are plotted as under:

\section{pH v/s Time (for five years)}

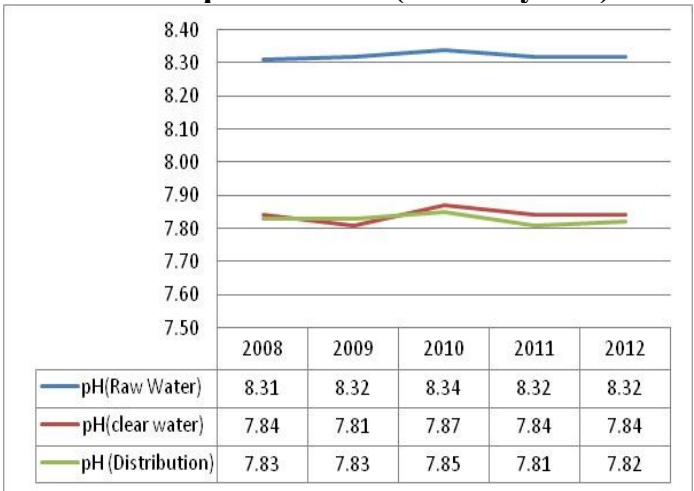

Acceptable Limit as per IS $=6.5-8.5$
Turbidity v/s Time (for five years)

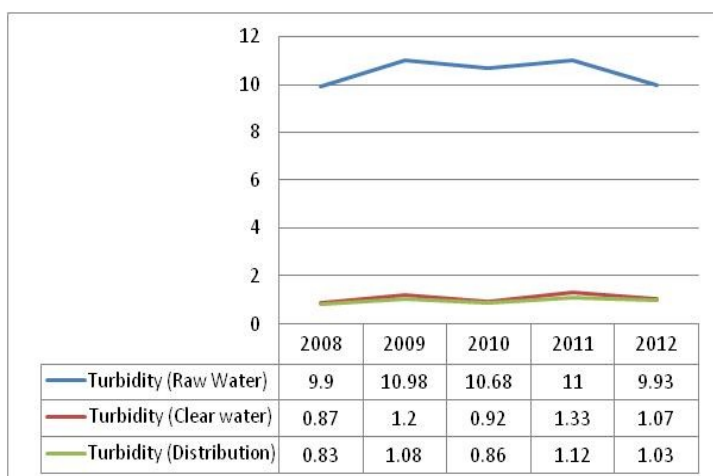

Acceptable Limit as per IS $=5(\mathrm{Max})$ 
Hardness v/s Time (for five years)

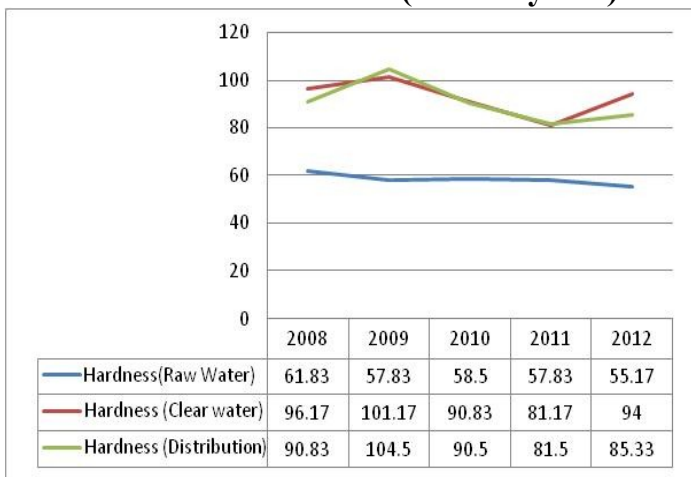

Acceptable limit as per IS=300 (Max)

TDS v/s Time (for five years)

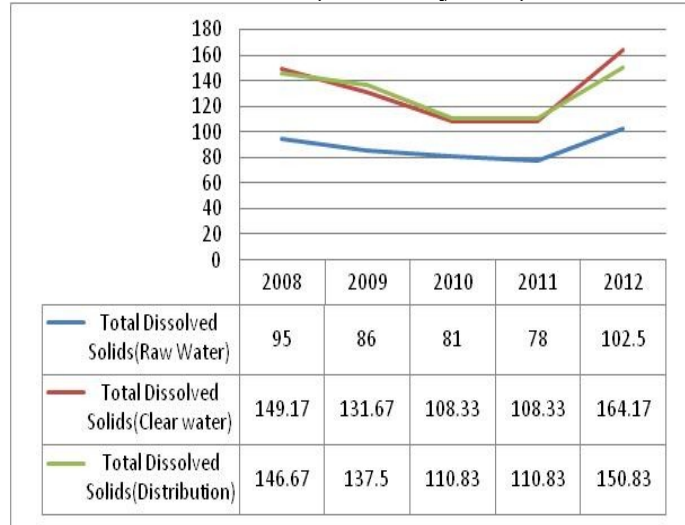

Acceptable limit as per IS=500 (Max)
Chloride v/s Time (for five years)

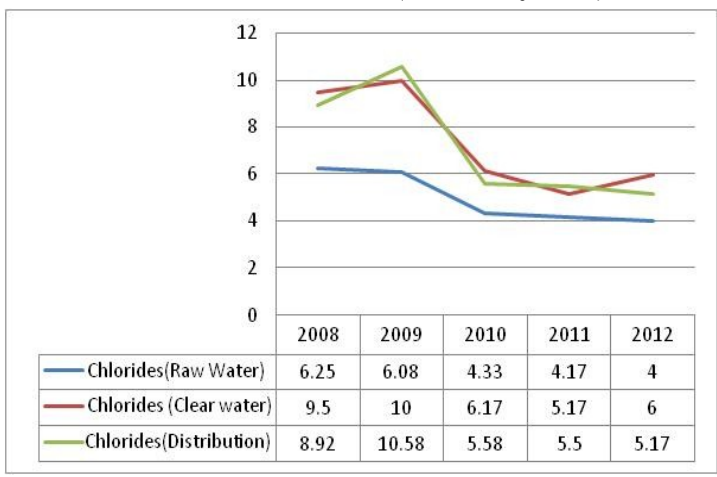

Acceptable limit as per IS=250 (Max)

FRC v/s Time (for five years)

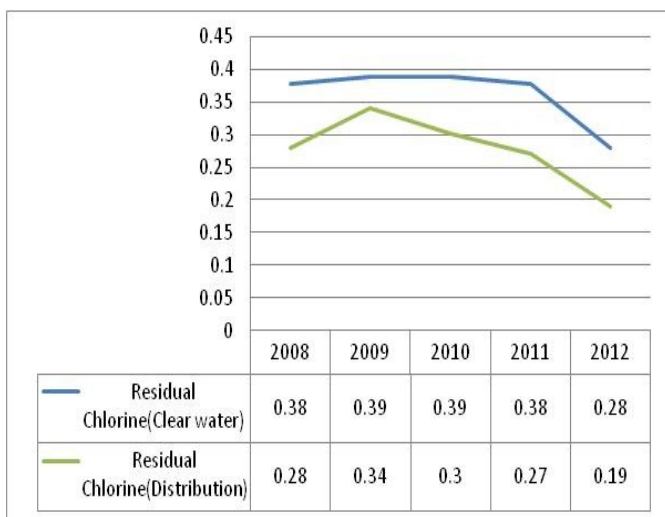

Acceptable limit as per IS $=0.2(\mathrm{Min})$

\section{Preparation of Chandigarh Hazard Analysis Matrix}

As per Module No 3 of WSP, all the possible visible \& hidden hazards that can contaminate the drinking water supplied to the City Chandigarh in the Pilot Study Zone No 2 have been looked into thoroughly at actual site right from Bhakra Catchment, raw water mains from Kajauli, in different stages of treatment process, treated water rising mains, distribution lines, consumer interface \& household storage \& handling practices.

The following 3 types of hazards have been looked into:

i. Physical Hazard (Denoted by $P$ in the Matrix)

ii. Chemical Hazard (Denoted by $C$ in the Matrix)

iii. Microbial Hazard (Denoted by $M$ in the matrix)

Any hazardous event can give rise to one, two or to all the three types of hazards.

The risk Matrix has been developed using semi quantitative approach.

The consequence or severity of a particular hazardous event has been rated from 1 to 5 started from the lowest impact to the highest impact.

$\begin{array}{lll}\text { Insignificant or no impact } & : & \text { Rating-1. } \\ \text { Minor impact } & : & \text { Rating-2 } \\ \text { Moderate impact } & : & \text { Rating-3 } \\ \text { Major impact } & : & \text { Rating-4 } \\ \text { Catastrophic impact } & : & \text { Rating-5 }\end{array}$

The likelihood or frequency of occurrence of a particular hazardous event has been rated from 1 to 5 starting from the lowest frequency to the highest frequency.
Rare/ Once every 5 years
Unlikely/ Once a year
Moderate/ once a month
Rating-1.
Likely/Once a week
: $\quad$ Rating-2
Almost/ certain once a day
Rating-4

Depending upon the severity and likelihood score/ rating, the overall risk for that particular hazardous event has been calculated as under: 


\section{Risk Score $=$ Severity rating $x$ Likelihood rating}

The risk score has been calculated as raw risks completely ignoring all the control measures that actually are in place in the water supply distribution system.

Depending upon the risk score, the risk band range for that particular hazardous event is taken in the following intervals in the hazard analysis matrix:

\section{Low (L): $\quad$ 1-5 (Denoted by Green Colour) \\ Medium (M) : 6-15 (Denoted by Yellow Colour) \\ High (H) : 16-25 (Denoted by Red Colour)}

As per Module No 4, the risk score is recalculated taking into account all the control measures that exist in the system to keep check on that particular hazardous event. So the severity and frequency of that hazardous event becomes low which ultimately brings down the risk score of that hazardous event.

This reassess risk has been incorporated in the Chandigarh Hazard Analysis Matrix to see whether the raw risk band has come down or not.

The complete Chandigarh Hazard Analysis Matrix as per Module No $3 \& 4$ has been prepared for all the possible hazardous events and is given in the next pages.

\begin{tabular}{|c|c|c|c|c|c|c|c|c|c|c|c|c|c|}
\hline No & $\begin{array}{l}\text { Source of } \\
\text { Risk } / \\
\text { Process } \\
\text { Step }\end{array}$ & Hazard & Hazardous Event & $\begin{array}{l}\text { Likeli } \\
\text { hood }\end{array}$ & $\begin{array}{l}\text { Conse } \\
\text { quence }\end{array}$ & $\begin{array}{l}\text { Risk Rating } \\
\text { or Raw Risk } \\
\text { (with no } \\
\text { controls) }\end{array}$ & $\begin{array}{l}\text { Risk } \\
\text { Band }\end{array}$ & $\begin{array}{l}\text { BASIS (Reasons } \\
\text { for selection of } \\
\text { likelihood \& } \\
\text { Consequence } \\
\text { Sources) }\end{array}$ & Control Measures & $\begin{array}{l}\text { Likeli } \\
\text { hood }\end{array}$ & $\begin{array}{l}\text { Conse } \\
\text { quence }\end{array}$ & $\begin{array}{c}\text { Risk } \\
\text { Rating or } \\
\text { Raw Risk } \\
\text { (with no } \\
\text { controls) }\end{array}$ & $\begin{array}{l}\text { Risk } \\
\text { Band }\end{array}$ \\
\hline \multirow[t]{7}{*}{1.} & $\begin{array}{c}\text { Bhakra } \\
\text { Catchment }\end{array}$ & M & $\begin{array}{l}\text { Sewage overflows from } \\
\text { human habitation during } \\
\text { monsoon period }\end{array}$ & 2 & 5 & 10 & M & $\begin{array}{l}\text { Human settlement } \\
\text { present near BML }\end{array}$ & $\begin{array}{l}\text { Septic tanks are in } \\
\text { place }\end{array}$ & 1 & 5 & 5 & $\mathrm{~L}$ \\
\hline & & $\mathrm{C}, \mathrm{P}, \mathrm{M}$ & $\begin{array}{c}\text { Idol immersion, Religious } \\
\text { activities }\end{array}$ & 2 & 4 & 8 & M & $\begin{array}{c}\text { There is no fencing } \\
\text { temple present close to } \\
\text { river }\end{array}$ & $\begin{array}{l}\text { Provision of separate } \\
\text { tanks for immersion }\end{array}$ & 1 & 4 & 4 & $\mathrm{~L}$ \\
\hline & & $\mathrm{C}, \mathrm{P}$ & $\begin{array}{l}\text { Agricultural activities in } \\
\text { the river bed }\end{array}$ & 3 & 4 & 12 & M & $\begin{array}{l}\text { Visual inspection } \\
\text { during visit }\end{array}$ & \begin{tabular}{c|} 
Agricultural \\
activities are \\
restricted in river bed
\end{tabular} & 1 & 4 & 4 & $\mathrm{~L}$ \\
\hline & & $\mathrm{C}, \mathrm{P}, \mathrm{M}$ & $\begin{array}{c}\text { Contaminants in storm } \\
\text { water runoff during } \\
\text { monsoon }\end{array}$ & 4 & 3 & 12 & M & $\begin{array}{l}\text { May happen in rainy } \\
\text { season due to storm } \\
\text { water runoff }\end{array}$ & $\begin{array}{c}\text { No current control } \\
\text { measures }\end{array}$ & 4 & 3 & 12 & M \\
\hline & & M & $\begin{array}{l}\text { Unauthorized access for } \\
\text { recreation (swimming, } \\
\text { fishing) }\end{array}$ & 2 & 1 & 2 & $\mathrm{~L}$ & There is no fencing & Security in place & 1 & 1 & 1 & $\mathrm{~L}$ \\
\hline & & $\mathrm{C}, \mathrm{P}, \mathrm{M}$ & $\begin{array}{c}\text { Growth of aquatic weeds } \\
\text { increasing nutrients in } \\
\text { BML } \\
\end{array}$ & 4 & 3 & 12 & M & $\begin{array}{l}\text { Aquatic weeds are } \\
\text { observed near bank }\end{array}$ & $\begin{array}{c}\text { Screens are provided } \\
\text { at inlet }\end{array}$ & 1 & 3 & 3 & $\mathrm{~L}$ \\
\hline & & $\mathrm{C}, \mathrm{P}$ & $\begin{array}{l}\text { Corrosion of screens, gates } \\
\text { and mechanical parts }\end{array}$ & 4 & 3 & 12 & M & $\begin{array}{c}\text { Observed during visit } \\
\text { to BML }\end{array}$ & $\begin{array}{c}\text { Periodic repair \& } \\
\text { maintenance } \\
\text { program }\end{array}$ & 1 & 3 & 3 & $\mathrm{~L}$ \\
\hline \multirow[t]{4}{*}{2.} & $\begin{array}{l}\text { Raw Water } \\
\text { Mains }\end{array}$ & $\mathrm{P}, \mathrm{M}$ & $\begin{array}{l}\text { Increased sediments load, } \\
\text { weeds and debris }\end{array}$ & 3 & 3 & 9 & M & $\begin{array}{l}\text { This phenomena is } \\
\text { observed during } \\
\text { monsoon season }\end{array}$ & $\begin{array}{l}\text { No current control } \\
\text { measures }\end{array}$ & 3 & 3 & 9 & M \\
\hline & & $\mathrm{P}, \mathrm{C}$ & $\begin{array}{l}\text { Failure of the sluice gates, } \\
\text { valves etc }\end{array}$ & 2 & 3 & 6 & M & $\begin{array}{c}\text { As per information } \\
\text { provided by MC } \\
\text { officers }\end{array}$ & $\begin{array}{l}\text { Periodic repair and } \\
\text { maintenance } \\
\text { program }\end{array}$ & 1 & 3 & 3 & $\mathrm{~L}$ \\
\hline & & P & $\begin{array}{c}\text { Lealcage in MS Pipe of } \\
\text { Phase I \& II }\end{array}$ & 1 & 5 & 5 & $\mathrm{~L}$ & $\begin{array}{c}\text { As per information } \\
\text { provided by MC } \\
\text { officers }\end{array}$ & $\begin{array}{l}\text { Immediate repair \& } \\
\text { Mtc programme (or } \\
\text { priority) }\end{array}$ & 1 & 5 & 5 & $\mathrm{~L}$ \\
\hline & & P & $\begin{array}{c}\text { Leakage in PSC Pipe of } \\
\text { Phase III \& IV }\end{array}$ & 3 & 5 & 15 & M & $\begin{array}{l}\text { As per information } \\
\text { provided by MC } \\
\text { officers }\end{array}$ & $\begin{array}{l}\text { Immediate repair \& } \\
\text { Mtc programme (or } \\
\text { priority) }\end{array}$ & 1 & 5 & 5 & $\mathrm{~L}$ \\
\hline 3. & $\begin{array}{c}\text { Treatment } \\
\text { plant }\end{array}$ & & & & & & & & & & & & \\
\hline \multirow[t]{2}{*}{$3 \mathrm{a}$} & $\begin{array}{l}\text { Chemical } \\
\text { dosing } \\
\text { system }\end{array}$ & $\mathrm{C}, \mathrm{P}$ & Over/ under dosing & 3 & 3 & 9 & M & $\begin{array}{l}\text { Dosing is monitored } \\
\text { daily }\end{array}$ & $\begin{array}{c}\text { Chemist determines } \\
\text { required dose by Jar } \\
\text { test daily. }\end{array}$ & 1 & 3 & 3 & $\mathrm{~L}$ \\
\hline & & $C, P$ & Mishandling of chemicals & 2 & 4 & 8 & M & $\begin{array}{l}\text { Visual inspection } \\
\text { during visit }\end{array}$ & $\begin{array}{c}\text { Standard operating } \\
\text { procedures are being } \\
\text { followed }\end{array}$ & 1 & 4 & 4 & $\mathrm{~L}$ \\
\hline
\end{tabular}




\begin{tabular}{|c|c|c|c|c|c|c|c|c|c|c|c|c|c|}
\hline No & $\begin{array}{l}\text { Source of } \\
\text { Risk } J \\
\text { Process } \\
\text { Step }\end{array}$ & Hazard & Hazardous Event & $\begin{array}{l}\text { Likeli } \\
\text { hood }\end{array}$ & $\begin{array}{l}\text { Conse } \\
\text { quence }\end{array}$ & $\begin{array}{l}\text { Risk Rating } \\
\text { or Raw Risk } \\
\text { (with no } \\
\text { controls) }\end{array}$ & $\begin{array}{c}\text { Risk } \\
\text { Band }\end{array}$ & $\begin{array}{l}\text { BASIS (Reasons } \\
\text { for selection of } \\
\text { likelihood \& } \\
\text { Consequence } \\
\text { Sources) }\end{array}$ & Control Measures & $\begin{array}{l}\text { Likeli } \\
\text { hood }\end{array}$ & $\begin{array}{l}\text { Conse } \\
\text { quence }\end{array}$ & $\begin{array}{l}\text { Risk } \\
\text { Rating or } \\
\text { Raw Risk } \\
\text { (with no } \\
\text { controls) }\end{array}$ & $\begin{array}{l}\text { Risk } \\
\text { Band }\end{array}$ \\
\hline \multirow[t]{2}{*}{$3 \mathrm{~b}$} & $\begin{array}{c}\text { Flash } \\
\text { mixing }\end{array}$ & $\mathrm{P}, \mathrm{C}$ & Electrical failure & 1 & 4 & 4 & $\mathrm{~L}$ & $\begin{array}{l}\text { Powver failure from } \\
\text { electricity board not } \\
\text { frequent }\end{array}$ & $\begin{array}{c}\text { Plant will shut down } \\
\text { automatically since } \\
\text { water cannot be } \\
\text { pumped. }\end{array}$ & 1 & 2 & 2 & L \\
\hline & & $\mathrm{C}, \mathrm{P}, \mathrm{M}$ & Improper settling & 3 & 4 & 12 & M & $\begin{array}{l}\text { If settler is } \\
\text { overloaded, or short } \\
\text { detention time }\end{array}$ & $\begin{array}{l}\text { Testing of Turbidity } \\
\text { after every } 2 \text { hours } \\
\text { and maintaining the } \\
\text { flow rate at inlet }\end{array}$ & 1 & 4 & 4 & $\mathrm{~L}$ \\
\hline \multirow[t]{3}{*}{$3 \mathrm{~d}$} & $\begin{array}{l}\text { Rapid Sand } \\
\text { Filtration }\end{array}$ & $\mathrm{C}, \mathrm{P}, \mathrm{M}$ & Improper filtration & 4 & 4 & 16 & $\mathrm{H}$ & $\begin{array}{l}\text { Online monitors for all } \\
\text { filters were found to } \\
\text { be not working }\end{array}$ & $\begin{array}{l}\text { Backwashing is done } \\
\text { depending on head } \\
\text { loss at each filter. } \\
\text { turbidity of filtered } \\
\text { water is tested once } \\
\text { in } 2 \text { hours }\end{array}$ & 1 & 4 & 4 & L \\
\hline & & $\mathrm{C}, \mathrm{P}, \mathrm{M}$ & $\begin{array}{c}\text { Loss of efficiency of filter } \\
\text { media }\end{array}$ & 2 & 4 & 8 & M & $\begin{array}{l}\text { Information given by } \\
\text { Plant Operator }\end{array}$ & $\begin{array}{l}\text { SOP's are developed } \\
\text { for Op \& Mtc of } \\
\text { filters }\end{array}$ & 1 & 4 & 4 & $\mathrm{~L}$ \\
\hline & & $\mathrm{C}, \mathrm{P}, \mathrm{M}$ & $\begin{array}{c}\text { Sand loss during back } \\
\text { wash resulting in reduction } \\
\text { of efficiency }\end{array}$ & 3 & 3 & 9 & M & $\begin{array}{c}\text { Reduced efficiency } \\
\text { may lead to improper } \\
\text { filtration }\end{array}$ & $\begin{array}{c}\text { Filter media is top up } \\
\text { on its loss }\end{array}$ & 1 & 3 & 3 & L \\
\hline \multirow[t]{4}{*}{$3 e$} & $\begin{array}{l}\text { Post-chlori- } \\
\text { nation }\end{array}$ & C & $\begin{array}{l}\text { Overdosing (formation of } \\
\text { DBP's, can exceed health } \\
\text { limits- } 5 \mathrm{mg} / \text { ) }\end{array}$ & 3 & 4 & 12 & M & $\begin{array}{l}\text { Based on water testing } \\
\text { records for residual } \\
\text { chlorine }\end{array}$ & $\begin{array}{l}\text { Online monitors are } \\
\text { present. Residual } \\
\text { chlorine is tested in } \\
\text { laboratory daily } \\
\text { every } 2 \text { hours }\end{array}$ & 1 & 4 & 4 & $\mathrm{~L}$ \\
\hline & & $\mathrm{M}$ & Under dosing & 3 & 5 & 15 & M & $\begin{array}{c}\text { Based on water testing } \\
\text { records for residual } \\
\text { chlorine and } \\
\text { bacteriological } \\
\text { analvsis }\end{array}$ & $\begin{array}{l}\text { Online monitors are } \\
\text { present }\end{array}$ & 1 & 5 & 5 & L \\
\hline & & M & $\begin{array}{l}\text { Unavailability of gas } \\
\text { cylinders }\end{array}$ & 1 & 4 & 4 & $\mathrm{~L}$ & $\begin{array}{l}\text { No such incident has } \\
\text { occurred in the } \\
\text { experience of the Plant } \\
\text { operator }\end{array}$ & $\begin{array}{c}\text { Gas cylinders are } \\
\text { always present in } \\
\text { spare }\end{array}$ & 1 & 4 & 4 & L \\
\hline & & M & Electrical failure & 4 & 4 & 16 & $\mathrm{H}$ & $\begin{array}{l}\text { Power fallure from } \\
\text { Elecy board not } \\
\text { frequent }\end{array}$ & Plant will shut down. & 2 & 2 & 4 & $\mathrm{~L}$ \\
\hline 4 & $\begin{array}{l}\text { Treated } \\
\text { Water } \\
\text { Rising } \\
\text { Mains } \\
\end{array}$ & $\mathrm{C}, \mathrm{P}, \mathrm{M}$ & $\begin{array}{l}\text { Microbial re growth, Taste } \\
\text { and Odour complaints due } \\
\text { to stripping of Bio-films }\end{array}$ & 3 & 5 & 15 & M & $\begin{array}{l}\text { Post chlorination at } \\
\text { treatment plants } \\
\text { reduces the } \\
\text { consequences } \\
\end{array}$ & $\begin{array}{l}\text { Periodic cleaning } \\
\text { and maintenance } \\
\text { program }\end{array}$ & 1 & 5 & 5 & $\mathrm{~L}$ \\
\hline \multirow[t]{4}{*}{ No } & $\begin{array}{l}\text { Source of } \\
\text { Riskly } \\
\text { Process } \\
\text { Step }\end{array}$ & Hazard & Hazardous Event & $\begin{array}{l}\text { L.ikeli } \\
\text { hood }\end{array}$ & $\begin{array}{l}\text { Conse } \\
\text { quence }\end{array}$ & $\begin{array}{l}\text { Risk Rating } \\
\text { or Raw Risk } \\
\text { (with no } \\
\text { controls) }\end{array}$ & $\begin{array}{c}\text { Risk } \\
\text { Band }\end{array}$ & $\begin{array}{l}\text { BASIS (Reasons } \\
\text { for selection of } \\
\text { likelihood \& } \\
\text { Consequence } \\
\text { Sources) }\end{array}$ & Control Measures & $\begin{array}{l}\text { L.jkeli } \\
\text { hood }\end{array}$ & $\begin{array}{l}\text { Conse } \\
\text { quemse. }\end{array}$ & $\begin{array}{c}\text { Risk } \\
\text { Rating or } \\
\text { Raw Risk } \\
\text { (with no } \\
\text { controls) }\end{array}$ & $\begin{array}{c}\text { Risk } \\
\text { Band }\end{array}$ \\
\hline & & $\mathrm{C}, \mathrm{P}, \mathrm{M}$ & $\begin{array}{l}\text { Contamination from pipe } \\
\text { bursts }\end{array}$ & 2 & 4 & 8 & M & $\begin{array}{l}\text { Thare is continuous } \\
\text { supply with high } \\
\text { presure }\end{array}$ & $\begin{array}{l}\text { Leak rapair program } \\
\text { on priority basis }\end{array}$ & 1 & 4 & 4 & L \\
\hline & & $\mathrm{C}, \mathrm{P}, \mathrm{M}$ & $\begin{array}{c}\text { Contamination from drains } \\
\text { during Mtte of pipes or } \\
\text { fittings }\end{array}$ & 2 & 5 & 10 & M & $\begin{array}{l}\text { As per difficulties } \\
\text { facsd by MC officials }\end{array}$ & $\begin{array}{l}\text { Super chlorination is } \\
\text { done after rapair }\end{array}$ & 1 & 5 & 5 & L \\
\hline & & $\mathrm{C}, \mathrm{P}$ & $\begin{array}{l}\text { Chemical contamination } \\
\text { dus to intemal corrosion }\end{array}$ & 2 & 4 & 8 & M & $\begin{array}{c}\text { Good quality materials } \\
\text { are usad. }\end{array}$ & $\begin{array}{l}\text { Periodic repair and } \\
\text { maintenance } \\
\text { activities are done }\end{array}$ & 1 & 4 & 4 & $\mathrm{~L}$ \\
\hline \multirow[t]{3}{*}{5} & & c & $\begin{array}{c}\text { High Fe levels in water } \\
\text { due to intemal conrosion of } \\
\text { unlingd Cast Iron mains }\end{array}$ & 4 & 2 & 8 & M & $\begin{array}{l}\text { Due to oxidation of } \mathrm{Fe} \\
\text { by chlonine in unlined } \\
\text { CI pipe network }\end{array}$ & $\begin{array}{l}\text { No current control } \\
\text { measuras }\end{array}$ & 4 & 2 & 8 & M \\
\hline & & $\mathrm{P}, \mathrm{M}$ & $\begin{array}{l}\text { Ingress of contaminants } \\
\text { due to leakage from } \\
\text { hydrants, fittings \& } \\
\text { perforations }\end{array}$ & 2 & 4 & 8 & M & Intenmittent supply & $\begin{array}{l}\text { Lakkage rapair } \\
\text { program }\end{array}$ & 1 & 4 & 4 & L \\
\hline & & $\mathrm{P}, \mathrm{M}$ & $\begin{array}{l}\text { Ingress of contaminants } \\
\text { from cossing of natural } \\
\text { drains nallaht }\end{array}$ & 3 & 5 & 15 & $M$ & $\begin{array}{l}\text { Control measures in } \\
\text { place, this problem } \\
\text { occurs only in } \\
\text { monsoon season when } \\
\text { drains are full }\end{array}$ & $\begin{array}{c}\text { Pipes are laid on } \\
\text { piers to avoid contact } \\
\text { with drains. }\end{array}$ & 2 & 5 & 10 & M \\
\hline \multirow[t]{6}{*}{6} & \begin{tabular}{|c|} 
Distri- \\
bution \\
mains (MS, \\
DI, CI) \\
\end{tabular} & $\mathrm{C}, \mathrm{P}, \mathrm{M}$ & $\begin{array}{l}\text { Contamination due to } \\
\text { ingress of foreign matter } \\
\text { from perforations in } \\
\text { corroded pipes }\end{array}$ & 3 & 5 & 15 & M & $\begin{array}{l}\text { Public Health } \\
\text { department records }\end{array}$ & $\begin{array}{l}\text { Existing visual } \\
\text { detection and repair } \\
\text { of laaks. }\end{array}$ & 2 & 5 & 10 & M \\
\hline & & $\mathrm{c}$ & $\begin{array}{c}\text { High Fe levels in water } \\
\text { due to intemal conrosion of } \\
\text { unlingd CI mains }\end{array}$ & 4 & 2 & 8 & M & $\begin{array}{l}\text { Due to oxidation of } \mathrm{Fe} \\
\text { by chlonine in unlined } \\
\text { CI pipe network }\end{array}$ & $\begin{array}{l}\text { No current control } \\
\text { measures }\end{array}$ & 4 & 2 & 8 & M \\
\hline & & c & $\begin{array}{l}\text { Potential of corrosion in } \\
\text { fittings }\end{array}$ & 3 & 2 & 6 & M & $\begin{array}{l}\text { Due to oxidation of } \mathrm{Fe} \\
\text { by chlorine in fittings }\end{array}$ & $\begin{array}{c}\text { No current control } \\
\text { measures }\end{array}$ & 3 & 2 & 6 & M \\
\hline & & $\mathrm{P}, \mathrm{M}$ & $\begin{array}{l}\text { Ingress of contaminants } \\
\text { due to tampering tapping } \\
\text { of maing }\end{array}$ & 4 & 4 & 16 & H & $\begin{array}{c}\text { Some cases } \\
\text { experienced by } \\
\text { officials of P.H Deptt }\end{array}$ & $\begin{array}{l}\text { Periodic inapection } \\
\text { and control }\end{array}$ & 2 & 4 & 8 & M \\
\hline & & $\mathrm{C}, \mathrm{P}, \mathrm{M}$ & $\begin{array}{l}\text { Ingrass of contaminants } \\
\text { from cossing of natural } \\
\text { drains pallahts }\end{array}$ & 3.5 & 5 & 17.5 & H & $\begin{array}{l}\text { Control measures in } \\
\text { place, this problem } \\
\text { occurs only in } \\
\text { monsoon season when } \\
\text { drains are full }\end{array}$ & $\begin{array}{l}\text { Pipes are laid on } \\
\text { piers piles to avoid } \\
\text { contact with drains. }\end{array}$ & 2 & 5 & 10 & M \\
\hline & & $\mathrm{P}, \mathrm{M}$ & $\begin{array}{c}\text { Contamination due to } \\
\text { ingress of foreign matter } \\
\text { from bursts accidental } \\
\text { breakage }\end{array}$ & 3.5 & 5 & 17.5 & н & $\begin{array}{c}\text { Public Health } \\
\text { department records }\end{array}$ & $\begin{array}{l}\text { Existing visual } \\
\text { detection and repair } \\
\text { of leaks. }\end{array}$ & 2 & 5 & 10 & M \\
\hline
\end{tabular}




\begin{tabular}{|c|c|c|c|c|c|c|c|c|c|c|c|c|c|}
\hline No & $\begin{array}{l}\text { Source of } \\
\text { Riskl } \\
\text { Process } \\
\text { Step }\end{array}$ & Hazard & Hazardous Event & $\begin{array}{l}\text { L.ikkeli } \\
\text { hood }\end{array}$ & $\begin{array}{l}\text { Conse } \\
\text { quence }\end{array}$ & $\begin{array}{l}\text { Risk Rating } \\
\text { or Raw Risk } \\
\text { (with no } \\
\text { controls) }\end{array}$ & $\begin{array}{l}\text { Risk } \\
\text { Band }\end{array}$ & $\begin{array}{l}\text { BASIS (Reasons } \\
\text { for selection of } \\
\text { likelihood \& } \\
\text { Consequence } \\
\text { Sources) }\end{array}$ & Control Measures & $\begin{array}{l}\text { L.jkeli } \\
\text { hood }\end{array}$ & $\begin{array}{l}\text { Conse } \\
\text { quence }\end{array}$ & $\begin{array}{c}\text { Risk } \\
\text { Rating or } \\
\text { Raw Risk } \\
\text { (with no } \\
\text { controls) }\end{array}$ & $\begin{array}{l}\text { Risk } \\
\text { Band }\end{array}$ \\
\hline & & $\mathrm{C}, \mathrm{P}$ & $\begin{array}{l}\text { Use of substandard } \\
\text { matenial for pipes }\end{array}$ & 3 & 4 & 12 & M & $\begin{array}{c}\text { Lead joints are usad in } \\
\text { old network, pipes are } \\
\text { prone to breakage }\end{array}$ & $\begin{array}{l}\text { Hydraulic testing is } \\
\text { done, check for ISI } \\
\text { mark, certificate } \\
\text { from manufacturer }\end{array}$ & 2 & 4 & 8 & M \\
\hline \multirow[t]{9}{*}{7} & $\begin{array}{l}\text { Consumer } \\
\text { interface }\end{array}$ & M,P & $\begin{array}{l}\text { Backflow from domestic } \\
\text { properties dus to absence } \\
\text { of chack valves }\end{array}$ & 4 & 5 & 20 & H & $\begin{array}{c}\text { Customer complaints } \\
\text { for contaminatsd } \\
\text { water } \\
\end{array}$ & $\begin{array}{l}\text { Periodic inspection } \\
\text { and control }\end{array}$ & 3 & 5 & 15 & M \\
\hline & & $\mathrm{P}, \mathrm{M}$ & $\begin{array}{l}\text { Intemal doss } \\
\text { contamination from sever } \\
\text { linas }\end{array}$ & 4 & 5 & 20 & н & $\begin{array}{l}\text { Customer complaints } \\
\text { and visual inspection }\end{array}$ & $\begin{array}{l}\text { No current control } \\
\text { measures }\end{array}$ & 4 & 5 & 20 & н \\
\hline & & $\mathrm{c}$ & $\begin{array}{l}\text { Corrosion of GI house } \\
\text { servica comnaction }\end{array}$ & 3 & 5 & 15 & M & $\begin{array}{l}\text { Maximum customer } \\
\text { complaints for } \\
\text { contaminated water } \\
\text { due to conroded } \\
\text { gervice connections } \\
\end{array}$ & $\begin{array}{c}\text { No current control } \\
\text { measures only pipe } \\
\text { from famule to meter } \\
\text { is charged on } \\
\text { conaumer's expense. }\end{array}$ & 2 & 5 & 10 & M \\
\hline & & $\mathrm{P}, \mathrm{M}$ & $\begin{array}{l}\text { Contamination in } \\
\text { sump/deep pits and } \\
\text { overhasd tanks vithin the } \\
\text { property }\end{array}$ & 5 & 5 & 25 & H & $\begin{array}{l}\text { Studies have shoun } \\
\text { the prevalence of } \\
\text { water-bome diseases }\end{array}$ & $\begin{array}{l}\text { Only after an event, } \\
\text { advise customers. } \\
\text { There is no curnent } \\
\text { control measure to } \\
\text { manage this, existing } \\
\text { regulation-sump } \\
\text { should be } 0.6 \mathrm{~m} \\
\text { above ground level. }\end{array}$ & 5 & 5 & 25 & H \\
\hline & & $\mathrm{P}, \mathrm{M}, \mathrm{C}$ & $\begin{array}{l}\text { Contamination due to } \\
\text { illegal pumps causing } \\
\text { pressure loss dounstream }\end{array}$ & 3 & 4 & 12 & M & Insufficient data & $\begin{array}{l}\text { Periodic chacking } \\
\text { and control }\end{array}$ & 2 & 4 & 8 & M \\
\hline & $\begin{array}{c}\text { House-hold } \\
\text { Storage }\end{array}$ & $\mathrm{P}, \mathrm{M}$ & $\begin{array}{l}\text { Storage vassel is designad } \\
\text { for dipping }\end{array}$ & 5 & 5 & 25 & H & $\begin{array}{l}\text { Visual inspection } \\
\text { during sanitary } \\
\text { surveillance }\end{array}$ & $\begin{array}{c}\text { Use of storage vessel } \\
\text { with tap }\end{array}$ & 4 & 5 & 20 & $\mathrm{H}$ \\
\hline & & $\mathrm{P}, \mathrm{M}$ & $\begin{array}{l}\text { Using dipper vith short or } \\
\text { no handle }\end{array}$ & 5 & 5 & 25 & H & $\begin{array}{l}\text { Visual inspection } \\
\text { during sanitary } \\
\text { surveillance }\end{array}$ & $\begin{array}{c}\text { Use of ladle with } \\
\text { long handle to access } \\
\text { water }\end{array}$ & 4 & 5 & 20 & $\mathrm{H}$ \\
\hline & & $\mathrm{P}, \mathrm{M}$ & $\begin{array}{l}\text { Vassel accessible to } \\
\text { children }\end{array}$ & 4 & 4 & 16 & H & $\begin{array}{l}\text { Discussion with } \\
\text { consumers during } \\
\text { sanitary surveillance }\end{array}$ & $\begin{array}{l}\text { Kesp vassels at } \\
\text { appropriate haight }\end{array}$ & 3 & 4 & 12 & M \\
\hline & & M & $\begin{array}{c}\text { Use pour back excess } \\
\text { water into the storage } \\
\text { vessel }\end{array}$ & 3 & 4 & 12 & M & $\begin{array}{l}\text { Discussion with } \\
\text { consumers during } \\
\text { sanitary survellance }\end{array}$ & $\begin{array}{c}\text { In some households, } \\
\text { excess water was not } \\
\text { poured back into } \\
\text { storage vessel but } \\
\text { was used for other } \\
\text { purposes } \\
\end{array}$ & 3 & 4 & 12 & M \\
\hline
\end{tabular}

\section{WSP Improvement Plans}

As per Module No 5, the improvement plans for the hazardous events which still remain on the higher side even after the control measures installed (as observed from the Chandigarh Hazard Analysis matrix) are being suggested in the proper format as per WSP Module. The improvement plans are also suggested to further lower the risk band even if already in control. The improvement plans clearly pin point the agencies responsible to execute that improvement in a clearly mentioned time frame.

\section{WSP Improvement Plan (Chandigarh City)}

\begin{tabular}{|c|c|c|c|c|c|}
\hline Process Step & Issue Identified & Improvements Required & Responsibility & $\begin{array}{l}\text { Time } \\
\text { Frame }\end{array}$ & Remarks \\
\hline \multicolumn{6}{|c|}{ BHAKRA CATCHMENT } \\
\hline & $\begin{array}{l}\text { Sewage Over - flow } \\
\text { from human } \\
\text { habitation during } \\
\text { monsoon period }\end{array}$ & $\begin{array}{l}\text { 1. Proper sewage collection, } \\
\text { treatment \& disposal in } \\
\text { catchment. } \\
\text { 2. No approval to habitations } \\
\text { without proper sewage } \\
\text { management. }\end{array}$ & $\begin{array}{l}\text { 1. Public Health } \\
\text { Department of MC } \\
\text { Chandigarh } \\
\text { 2. Local NGOs }\end{array}$ & 3 years & $\begin{array}{l}\text { As catchment area } \\
\text { is not under MC } \\
\text { Chd \& } \\
\text { correspondence } \\
\text { will be done with } \\
\text { authorities of } \\
\text { nearby habitations }\end{array}$ \\
\hline & Human Defection & $\begin{array}{l}\text { 1. Strictly prohibiting. } \\
\text { Unauthorized access. } \\
\text { 2. Awareness programs to } \\
\text { educate people about proper } \\
\text { sanitary practices. }\end{array}$ & $\begin{array}{l}\text { 1. Public Health } \\
\text { Department of MC } \\
\text { Chandigarh } \\
\text { 2. Local NGOs }\end{array}$ & Uncertain & $\begin{array}{l}\text { Awareness } \\
\text { programs will be } \\
\text { carried out }\end{array}$ \\
\hline & $\begin{array}{l}\text { Contamination due } \\
\text { to idol immersion } \\
\text { and other religious } \\
\text { activities. }\end{array}$ & $\begin{array}{l}\text { 1. Protect streams connected to } \\
\text { reservoir in catchment. } \\
\text { 2. Prohibit idol immersion } \\
\text { along with other religious } \\
\text { activities in these streams. } \\
\text { 3. Awareness programs to } \\
\text { educate people about possible } \\
\text { Water contamination \& their } \\
\text { health effects. } \\
\text { 4.Provide separate dedicated } \\
\text { pond for idol immersion. }\end{array}$ & $\begin{array}{l}\text { 1. Public Health } \\
\text { Department of MC } \\
\text { Chandigarh } \\
\text { 2. Local NGOs }\end{array}$ & Uncertain & $\begin{array}{l}\text { Awareness } \\
\text { programs will be } \\
\text { carried out }\end{array}$ \\
\hline & $\begin{array}{c}\text { Agricultural } \\
\text { activities in the river } \\
\text { bed }\end{array}$ & $\begin{array}{l}\text { Agricultural activities in the } \\
\text { river bed should be restricted }\end{array}$ & $\begin{array}{l}\text { 1. Health \& Sanitation } \\
\text { Deptt. } \\
\text { 2. D.C. Morinda }\end{array}$ & 1 year & $\begin{array}{l}\text { Major agricultural } \\
\text { activities in the } \\
\text { river bed are } \\
\text { already restricted }\end{array}$ \\
\hline
\end{tabular}


A Study of Water Safety Plan (WSP) For Environmental Risk Management of a Modern North ....

\begin{tabular}{|c|c|c|c|c|c|}
\hline & $\begin{array}{l}\text { Contaminants in } \\
\text { storm water runoff } \\
\text { during monsoon }\end{array}$ & $\begin{array}{l}\text { Ensure plant cover around the } \\
\text { reservoir }\end{array}$ & Forest Deptt. & Uncertain & \\
\hline & $\begin{array}{l}\text { Corrosion of } \\
\text { screens, gates and } \\
\text { mechanical parts }\end{array}$ & $\begin{array}{l}\text { Screens, gates, \& valves } \\
\text { should be replaced \&their } \\
\text { proper cleaning \& Mtc should } \\
\text { be ensured }\end{array}$ & $\begin{array}{l}\text { Public Health Deptt of } \\
\text { MC Chd }\end{array}$ & 1 year & \\
\hline \multicolumn{6}{|c|}{ RAW WATER MAINS } \\
\hline & $\begin{array}{l}\text { Increased sediment } \\
\text { load, weeds and } \\
\text { debris }\end{array}$ & $\begin{array}{l}\text { Screens should be installed at } \\
\text { intake \& these should be } \\
\text { properly cleaned \& maintained }\end{array}$ & $\begin{array}{l}\text { Public health Deptt of } \\
\text { M.C. Chandigarh }\end{array}$ & 1 year & \\
\hline & Growth of Bio film & $\begin{array}{l}\text { Periodic cleaning of mains } \\
\text { should be done }\end{array}$ & $\begin{array}{l}\text { Public health Deptt of } \\
\text { M.C. Chandigarh }\end{array}$ & 1 year & \\
\hline \multicolumn{6}{|c|}{ WATER TREATMENT PLANT WATER WORKS SECTOR 12} \\
\hline \multirow[t]{3}{*}{ Inlet } & $\begin{array}{l}\text { Floating debris, } \\
\text { grass in raw water }\end{array}$ & $\begin{array}{l}\text { Inlet screens should be } \\
\text { installed to prevent debris \& } \\
\text { grass from entering at inlet }\end{array}$ & $\begin{array}{l}\text { Public health Deptt of } \\
\text { M.C. Chandigarh }\end{array}$ & & \\
\hline & $\begin{array}{c}\text { Hydraulic } \\
\text { overloading at Inlet } \\
\text { chamber }\end{array}$ & $\begin{array}{l}\text { New flow measuring devices } \\
\text { should be installed and } \\
\text { properly calibrated }\end{array}$ & $\begin{array}{l}\text { Public health Deptt of } \\
\text { M.C. Chandigarh }\end{array}$ & & \\
\hline & $\begin{array}{l}\text { Leakage in inlet } \\
\text { valve }\end{array}$ & $\begin{array}{l}\text { Leak repair programme is } \\
\text { needed }\end{array}$ & $\begin{array}{l}\text { Public health Deptt of } \\
\text { M.C. Chandigarh }\end{array}$ & & \\
\hline $\begin{array}{l}\text { Pre- } \\
\text { chlorination }\end{array}$ & $\begin{array}{l}\text { Leakage in chlorine } \\
\text { pipe leading to } \\
\text { ineffective pre- } \\
\text { treatment } \\
\end{array}$ & $\begin{array}{l}\text { Pipe should be repaired and } \\
\text { chemical dosing should be } \\
\text { monitored }\end{array}$ & $\begin{array}{l}\text { Public health Deptt of } \\
\text { M.C. Chandigarh }\end{array}$ & & \\
\hline \multirow[t]{3}{*}{ PAC dosing } & Under/ over dosing & $\begin{array}{l}\text { Regular inspection should be } \\
\text { done, automatic dosing system } \\
\text { should be installed }\end{array}$ & $\begin{array}{l}\text { Public health Deptt of } \\
\text { M.C. Chandigarh }\end{array}$ & & \\
\hline & $\begin{array}{l}\text { Mishandling of } \\
\text { chemicals }\end{array}$ & $\begin{array}{l}\text { SOP's should be followed for } \\
\text { storage \& handling of } \\
\text { coagulant }\end{array}$ & $\begin{array}{l}\text { Public health Deptt of } \\
\text { M.C. Chandigarh }\end{array}$ & & \\
\hline & $\begin{array}{l}\text { Algal growth in } \\
\text { open channel }\end{array}$ & $\begin{array}{l}\text { Adequate pre-chlorination } \\
\text { should be done }\end{array}$ & $\begin{array}{l}\text { Public health Deptt of } \\
\text { M.C. Chandigarh }\end{array}$ & & \\
\hline \multirow[t]{2}{*}{ Flash mixing } & $\begin{array}{l}\text { Mechanical failure } \\
\text { results in improper } \\
\text { mixing }\end{array}$ & $\begin{array}{l}\text { Motor and agitator blades } \\
\text { should be replaced }\end{array}$ & $\begin{array}{l}\text { Public health Deptt of } \\
\text { M.C. Chandigarh }\end{array}$ & & \\
\hline & Electrical failure & $\begin{array}{l}\text { Electricity Board should } \\
\text { ensure uninterrupted power } \\
\text { supply to treatment plants }\end{array}$ & $\begin{array}{l}\text { Public health Deptt of } \\
\text { M.C. Chandigarh }\end{array}$ & & \\
\hline \multirow[t]{2}{*}{ Sedimentation } & Improper settling & $\begin{array}{l}\text { Settling tank should be cleaned } \\
\text { periodically, growth of aquatic } \\
\text { weeds and plants should be } \\
\text { checked }\end{array}$ & $\begin{array}{l}\text { Public health Deptt of } \\
\text { M.C. Chandigarh }\end{array}$ & & \\
\hline & $\begin{array}{c}\text { Improper de- } \\
\text { sludging }\end{array}$ & $\begin{array}{l}\text { Periodic de-sludging should be } \\
\text { done }\end{array}$ & $\begin{array}{l}\text { Public health Deptt of } \\
\text { M.C. Chandigarh }\end{array}$ & & \\
\hline \multirow[t]{2}{*}{$\begin{array}{l}\text { Rapid sand } \\
\text { filters }\end{array}$} & $\begin{array}{l}\text { Loss of efficiency of } \\
\text { filter media }\end{array}$ & $\begin{array}{l}\text { Complete over hauling of all } \\
12 \text { filters \& replacement of } \\
\text { filter media }\end{array}$ & M.C. Chandigarh & & \\
\hline & $\begin{array}{l}\text { Inefficient backwash } \\
\text { cycle }\end{array}$ & $\begin{array}{l}\text { Well-defined pressure drop/ } \\
\text { head loss \& continuous online } \\
\text { monitoring of pressure } \\
\text { differential should be done. } \\
\text { Standard operating procedures } \\
\text { for back washing should be } \\
\text { followed. Shut down of filters } \\
\text { when pressure drop is less than } \\
1.5 \mathrm{~m} \& \text { turbidity standards are } \\
\text { exceeded }\end{array}$ & M.C. Chandigarh & & \\
\hline \multirow[t]{5}{*}{$\begin{array}{l}\text { Post } \\
\text { Chlorination }\end{array}$} & Under dosing & $\begin{array}{l}\text { Chlorine demand should be } \\
\text { checked daily and flow rate } \\
\text { should be maintained }\end{array}$ & $\begin{array}{l}\text { Public health Deptt of } \\
\text { M.C. Chandigarh }\end{array}$ & & \\
\hline & Electrical failure & $\begin{array}{l}\text { Plant should be shut down } \\
\text { Provision for closing inlet } \\
\text { valves within seconds should } \\
\text { be made }\end{array}$ & $\begin{array}{l}\text { Public health Deptt of } \\
\text { M.C. Chandigarh }\end{array}$ & & \\
\hline & \multicolumn{4}{|c|}{ U.G.R./s } & \\
\hline & $\begin{array}{l}\text { Contamination due } \\
\text { to inspection } \\
\text { chambers not } \\
\text { properly covered } \\
\end{array}$ & $\begin{array}{l}\text { Daily inspection by responsible } \\
\text { person should be done; strict } \\
\text { instructions to the valve } \\
\text { operator should be given }\end{array}$ & $\begin{array}{l}\text { Public health Deptt of } \\
\text { M.C. Chandigarh }\end{array}$ & 6 month & \\
\hline & $\begin{array}{l}\text { Corrosion of ladder } \\
\text { due to contact with }\end{array}$ & $\begin{array}{l}\text { Non-corrosive for e.g. plastic } \\
\text { coated ladders should be used }\end{array}$ & $\begin{array}{l}\text { Public health Deptt of } \\
\text { M.C. Chandigarh }\end{array}$ & 6 month & \\
\hline
\end{tabular}


A Study of Water Safety Plan (WSP) For Environmental Risk Management of a Modern North ....

\begin{tabular}{|c|c|c|c|c|c|}
\hline & chlorine & & & & \\
\hline & $\begin{array}{l}\text { Security breach at } \\
\text { W/W Sec } 12\end{array}$ & $\begin{array}{l}\text { Strict security measures should } \\
\text { be kept in place }\end{array}$ & $\begin{array}{l}\text { Public health Deptt of } \\
\text { M.C. Chandigarh }\end{array}$ & 3 month & \\
\hline \multicolumn{6}{|c|}{ DISTRIBUTION MAINS } \\
\hline & $\begin{array}{l}\text { Contamination due } \\
\text { to ingress of foreign } \\
\text { matter from } \\
\text { perforations in } \\
\text { corroded pipes }\end{array}$ & $\begin{array}{l}\text { Use of non-corrosive pipes, } \\
\text { limited number of joins }\end{array}$ & $\begin{array}{l}\text { Public health Deptt of } \\
\text { M.C. Chandigarh }\end{array}$ & 2 years & $\begin{array}{l}\text { The rehabilitation } \\
\text { program for } \\
\text { improvement of } \\
\text { water distribution } \\
\text { is in the process }\end{array}$ \\
\hline & $\begin{array}{l}\text { Potential backflow } \\
\text { from tappings into } \\
\text { mains due to } \\
\text { absence of backflow } \\
\text { prevention devices }\end{array}$ & $\begin{array}{l}\text { Non returning valves should be } \\
\text { installed. }\end{array}$ & $\begin{array}{l}\text { Public health Deptt of } \\
\text { M.C. Chandigarh }\end{array}$ & 3 years & \\
\hline & $\begin{array}{l}\text { Potential backflow } \\
\text { from illegal tapings } \\
\text { into mains }\end{array}$ & $\begin{array}{l}\text { Strict action should be taken to } \\
\text { eliminate illegal tappings into } \\
\text { mains. Regular inspection } \\
\text { program should be carried out. }\end{array}$ & M.C. Chandigarh & 3 years & \\
\hline & $\begin{array}{l}\text { Biofilm formation } \\
\text { due to lack of } \\
\text { chlorine }\end{array}$ & $\begin{array}{l}\text { A schedule for routine } \\
\text { monitoring of chlorine should } \\
\text { be done at specified points in } \\
\text { distribution system. }\end{array}$ & M.C. Chandigarh & 1 year & \\
\hline \multicolumn{6}{|c|}{ CONSUMER INTERFACE } \\
\hline & $\begin{array}{l}\text { Contamination in } \\
\text { open sump/deep pits } \\
\text { within the property. }\end{array}$ & $\begin{array}{l}\text { 1. Measures should be taken } \\
\text { for periodic cleaning of } \\
\text { sumps/deep pits and overhead } \\
\text { tanks by MC. } \\
\text { 2. MC to develop \& carry out } \\
\text { awareness programs e.g. radio } \\
\text { \& television announcements, } \\
\text { posters. }\end{array}$ & M.C. Chandigarh & 3 years & \\
\hline & $\begin{array}{l}\text { Backflow from } \\
\text { domestic properties } \\
\text { due to absence of } \\
\text { check valves }\end{array}$ & $\begin{array}{l}\text { Installation of non-returning } \\
\text { valves }\end{array}$ & $\begin{array}{lc}\text { Water } & \text { Works } \\
\text { Department } & \text { M.C. } \\
\text { Chandigarh } & \end{array}$ & 5 years & \\
\hline & $\begin{array}{c}\text { Internal cross } \\
\text { contamination from } \\
\text { sewer lines }\end{array}$ & $\begin{array}{l}\text { Laying of pipe network should } \\
\text { be as per norms \& Non return } \\
\text { valves should be fitted }\end{array}$ & $\begin{array}{l}\text { Water Works } \\
\text { Department, MC \& } \\
\text { Sanitation Deptt, M.C. } \\
\text { Chandigarh }\end{array}$ & 2 year & \\
\hline & $\begin{array}{l}\text { Corrosion of GI } \\
\text { house service } \\
\text { connection }\end{array}$ & $\begin{array}{l}\text { Suggest customers to replace } \\
\text { existing GI pipes by non- } \\
\text { corrosive MDPE pipes }\end{array}$ & $\begin{array}{l}\text { 1. Water } \quad \text { Works } \\
\text { Department, } \\
\text { Chandigarh }\end{array}$ & Uncertain & $\begin{array}{l}\text { As it depends on } \\
\text { the financial status } \\
\text { as well as willing } \\
\text { of consumers }\end{array}$ \\
\hline & $\begin{array}{l}\text { Digging pit outside } \\
\text { the house during low } \\
\text { pressure }\end{array}$ & $\begin{array}{l}\text { Regular inspection should be } \\
\text { done and } 24 \times 7 \text { supply will } \\
\text { prevent low pressure }\end{array}$ & $\begin{array}{lr}\text { Water } & \text { Works } \\
\text { Department } & \text { M.C. } \\
\text { Chandigarh } & \\
\end{array}$ & 6 years & \\
\hline & $\begin{array}{l}\text { Contamination due } \\
\text { to illegal pumps } \\
\text { causing pressure } \\
\text { loss downstream } \\
\end{array}$ & $\begin{array}{l}\text { Legal action should be taken } \\
\text { against those who install illegal } \\
\text { pumps }\end{array}$ & $\begin{array}{lr}\text { Water } & \text { Works } \\
\text { Department } & \text { M.C. } \\
\text { Chandigarh } & \end{array}$ & 2 year & \\
\hline & $\begin{array}{l}\text { Over flow of storm } \\
\text { water drain near } \\
\text { consumer } \\
\text { connection }\end{array}$ & $\begin{array}{l}\text { Separate storm water drains } \\
\text { should be laid to prevent } \\
\text { overflow in sewer. }\end{array}$ & $\begin{array}{lr}\text { 1. Water } & \text { Works } \\
\text { Department, MC and } \\
\text { Health \& } & \text { Sanitation } \\
\text { Department } & \text { M.C. } \\
\text { Chandigarh } & \\
\text { 2. Consumer } & \end{array}$ & Uncertain & $\begin{array}{l}\text { As it depends on } \\
\text { the financial status } \\
\text { as well as willing } \\
\text { of consumers. }\end{array}$ \\
\hline \multicolumn{6}{|c|}{ HOUSEHOLD STORAGE } \\
\hline & $\begin{array}{c}\text { Storage vessels } \\
\text { designed for dipping }\end{array}$ & $\begin{array}{l}\text { Consumers should be made } \\
\text { aware about the advantages of } \\
\text { using vessels with a narrow } \\
\text { mouth and a tap }\end{array}$ & $\begin{array}{l}\text { 1. M.C. Chandigarh } \\
\text { 2. Local NGOs } \\
\text { 3. Local Media }\end{array}$ & 1 Year & \\
\hline & $\begin{array}{l}\text { Using dipper with } \\
\text { short or no handle }\end{array}$ & $\begin{array}{l}\text { Ladle with long handle should } \\
\text { be used to take out water from } \\
\text { vessel }\end{array}$ & $\begin{array}{l}\text { 1. M.C. Chandigarh } \\
\text { 2. Local NGOs } \\
\text { 3. Local Media }\end{array}$ & 1 year & \\
\hline & $\begin{array}{l}\text { Vessel accessible to } \\
\text { children }\end{array}$ & $\begin{array}{l}\text { Consumers should be made } \\
\text { aware of risks associated with } \\
\text { lack of hygiene. Children } \\
\text { should be discouraged from } \\
\text { accessing the storage vessels. }\end{array}$ & $\begin{array}{l}\text { 1. M.C. Chandigarh } \\
\text { 2. Local NGOs } \\
\text { 3. Local Media }\end{array}$ & 1 year & \\
\hline & $\begin{array}{l}\text { Users pour back } \\
\text { excess water into the } \\
\text { storage vessel }\end{array}$ & $\begin{array}{l}\text { Consumers should be made } \\
\text { aware about the risks of } \\
\text { unsanitary drinking water }\end{array}$ & $\begin{array}{l}\text { 1. M.C. Chandigarh } \\
\text { 2. Local NGOs } \\
\text { 3. Local Media }\end{array}$ & 1 year & \\
\hline
\end{tabular}




\begin{tabular}{|l|c|l|l|l|}
\hline & practices & & \\
\hline & $\begin{array}{l}\text { Vessel is made on } \\
\text { non-durable material }\end{array}$ & $\begin{array}{l}\text { Consumers should be } \\
\text { encouraged to use steel or } \\
\text { plastic vessels }\end{array}$ & $\begin{array}{l}\text { 1. M.C. Chandigarh } \\
\text { 2. Local NGOs } \\
\text { 3. Local Media }\end{array}$ & 1 year \\
\hline $\begin{array}{c}\text { Handling of } \\
\text { drinking water } \\
\text { without washing } \\
\text { hands }\end{array}$ & $\begin{array}{l}\text { Consumers should be made } \\
\text { aware about risks of unsanitary } \\
\text { drinking water practices \& } \\
\text { importance of personal \& } \\
\text { domestic hygiene }\end{array}$ & $\begin{array}{l}\text { 2. Local NGOs } \\
\text { 3. Local Media }\end{array}$ & 1 year \\
\hline
\end{tabular}

\section{Monitoring of Control Measures}

As per Module No 6, the monitoring requirement and corrective actions have been described for the raw water, treatment plant, UGR and consumer interface in the prescribed format under the Module.

All the critical limits for the different parameters at designated points have been described in the format. When it will be monitored, how it will be monitored and who will monitor it has been clearly stated in the said format. What corrective actions need to be taken in case the critical limit for that particular parameter is exceeded is also shown in the format.

Monitoring Requirements \& Corrective Actions

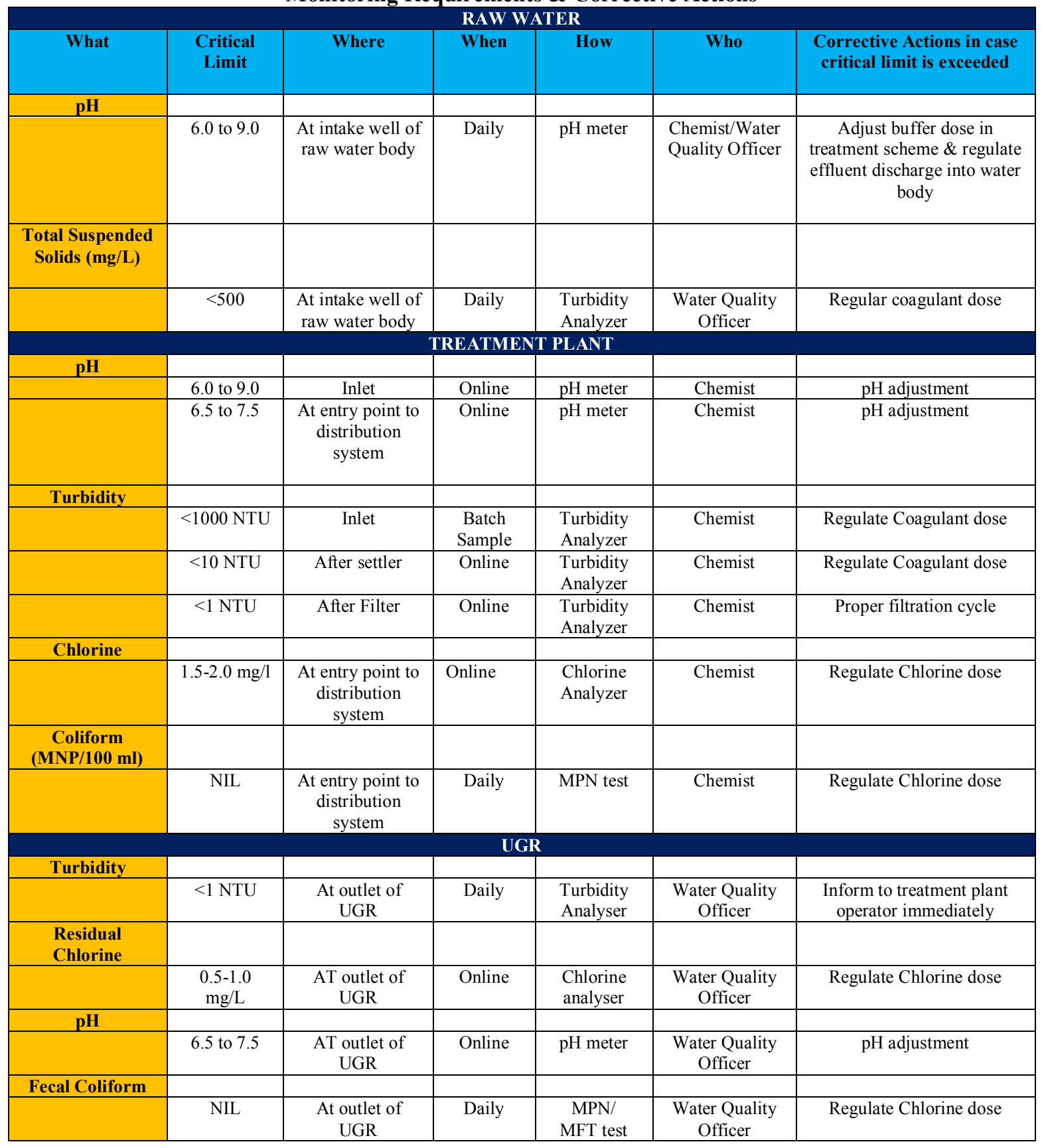




\begin{tabular}{|c|c|c|c|c|c|c|}
\hline \multicolumn{7}{|c|}{ CONSUMER INTERFACE } \\
\hline & $<1 \mathrm{NTU}$ & $\begin{array}{c}\text { At randomly } \\
\text { selected } \\
\text { representative } \\
\text { consumer taps }\end{array}$ & Daily & $\begin{array}{l}\text { Turbidity } \\
\text { Analyzer }\end{array}$ & $\begin{array}{l}\text { Water Quality } \\
\text { Officer }\end{array}$ & $\begin{array}{c}\text { Inform to Service Engineer } \\
\text { at ESR immediately }\end{array}$ \\
\hline \multicolumn{7}{|l|}{$\begin{array}{l}\text { Residual } \\
\text { Chlorine }\end{array}$} \\
\hline & $\begin{array}{c}0.2-0.5 \\
\mathrm{mg} / \mathrm{L}\end{array}$ & $\begin{array}{c}\text { At randomly } \\
\text { selected } \\
\text { representative } \\
\text { consumer taps }\end{array}$ & Daily & $\begin{array}{l}\text { Chlorine } \\
\text { Analyzer }\end{array}$ & $\begin{array}{l}\text { Water Quality } \\
\text { Officer }\end{array}$ & Regulate Chlorine dose \\
\hline \multicolumn{7}{|l|}{ Fecal Coliform } \\
\hline & NIL & $\begin{array}{c}\text { At randomly } \\
\text { selected } \\
\text { representative } \\
\text { consumer taps }\end{array}$ & Daily & $\begin{array}{c}\text { MPN/ } \\
\text { MFT test }\end{array}$ & $\begin{array}{l}\text { Water Quality } \\
\text { Officer }\end{array}$ & Regulate Chlorine dose \\
\hline \multicolumn{7}{|l|}{ pH } \\
\hline & 6.5 to 7.5 & $\begin{array}{c}\text { At randomly } \\
\text { selected } \\
\text { representative } \\
\text { consumer taps }\end{array}$ & Daily & $\mathrm{pH}$ meter & $\begin{array}{l}\text { Water Quality } \\
\text { Officer }\end{array}$ & $\mathrm{pH}$ adjustment \\
\hline
\end{tabular}

\section{Conclusion}

In the present study, the semi-quantitative risk matrix approach has been used for the development of Chandigarh Hazard analysis matrix considering the raw risks without any control measures and re-assessed risks with control measures in place. In case the re-assessed risk is still on a higher side, improvement and modification plans have also been suggested. Standard operating procedures in case critical limits of various parameters go out of control have also been developed.

The study area taken in the scope of this thesis is one of the six zones in which whole of the Chandigarh city has been divided for water supply distribution. In the present thesis, WSP has been developed for 1 zone only i.e. Pilot zone No. 2. This concept is applicable to any model city like Chandigarh. At the start, it is advisable to develop and implement the WSP in a Pilot area of the city and then depending upon its outcome / success, it can be extended to whole of the city.

The present study will also certainly be helpful in giving guidance to the WSP team selected for developing and implementing water safety plan for any other modern north Indian city like Chandigarh.

\section{References}

[1]. Adam M. Finkel \& John S. Evans (1987). Evaluating the Benefits of Uncertainty Reduction in Environmental Health Risk Management. JAPCA, Vol. 37, Issue 10, 1987, Pages 1164-1171

[2]. Ainswarth R. ed (2004). Safe piped water: Managing microbial water quality in piped distribution systems. World Health Organization, Geneva.

[3]. Arora H (1998). Application of particle counting to enhance water treatment operations. Voorhees, NJ, American water works service company, Inc.

[4]. $\mathrm{Au} \mathrm{K-K}$ et al. (2002). The role of oxidants on particle removal. Proceedings of the American Water Works Association Annual Conference.

[5]. Au K-K, Le Chavallier MW (2000). Effects of oxidation on particle removal: The role of natural organic matter. Proceedings of the American Water Works Association Water Quality Technology Conference.

[6]. AUSAID (2005). Safe water guide for the Australian and Programme 2005, A frame work and guidance for managing water quality. Published by the Australian Agency for International Development (AUSAID), Canberra.

[7]. AWWA (1979). Committee report: Viruses in drinking water. Journal of the American water works Association.

[8]. Bartram J, Balance R, eds. (1996). Water quality monitoring: a practical guide to the design \& implementation of fresh water quality standards \& monitoring programme, published on behalf of UNESCO, WHO \& UNEP by E\&FN Spon London.

[9]. Bartram J et al., eds. (2003), Heterotrophic plate count measurement and drinking water safety: The significance of HPCs for water quality and human health. World Health Organization, Geneva, IWA Publishing.

[10]. Bartram J, Corrales L, Davison A, Deere D, Drury D, Gordon B, Howard G, Reinhold A, Stevens M. (2009). Water Safety Plan Manual: step-by-step risk management for drinking-water suppliers, World Health Organization, Geneva, http://www.wsportal.org/ibis/water-safety-portal/eng/ home.

[11]. Bellamy WD et al. (1993), Assessing treatment plant performance. Journal of the American water works Association.

[12]. BGS (British Geological Survey) (2001), Assessing risk to groundwater from on-site sanitation, http://bgs.uk/hydrogeology/argoss.

[13]. Black, M. (1998) Learning what works: a 20 year retrospective view on international water and sanitation cooperation, 1978-1998, UNDP-World Bank Water and Sanitation Programme, Washington.

[14]. BPSU (2011) Bangladesh Local Government Division, National Guidelines on water safety framework in Bangladesh published by policy supply unit of Local Government Division, Ministry of Local Government, Rural Development Cooperates.

[15]. Brinduşa Mihaela Robu, Florentina Anca Căliman, Camelia Beţianu, Maria Gavrilescu (2007), Methods \& procedures for Environmental Risk Assessment, Environmental Engineering and Management Journal, Vol 6, No 6, Nov/ Dec 2007, Pages 573592 .

[16]. Bryan JJ (1993). Hazard analysis and critical control points and their applications to the drinking water process. American water works Association water quality technology Conference. Denver, CO, American water works Association. 
[17]. Bourbigot MM, Dodin A, Lheritier R (1984). Bacteria in distribution systems. Water Research.

[18]. Cairncross (1992), S. Sanitation and water supply practical lessons from the decade, UMDA-World Bank Water \& Sanitation Programme, Washington.

[19]. Camper AK et al. (1991). Growth kinetics of Coliform under conditions relevant to drinking water distribution system. Applied and Environmental Microbiology.

[20]. Chang SL (1982), The safety of water disinfection. Annual Review of Public Health.

[21]. Charles Vlek, Gideon Keren (1992). Behavioral decision theory \& environmental risk management: Assessment \& resolution of four 'survival' dilemmas. Acta Psychlogica, Vol. 80, Issues 1-3, August 1992, Pages 249-278.

[22]. Davison A et al (2005), Water safety plans, managing drinking water quality from catchment to consumers, water sanitation and health, World Health Organization, Geneva (WHO/SDE/WSH/05.06).

[23]. Davis EM, Casserly DM, Moore JD (1977). Bacterial relationships in storm waters, Water Resources Bulletin.

[24]. Deb AK (2004). Guidance for management of distribution system operation and maintenance. Denver, CO, American water works Association Research Foundation and American water works Association.

[25]. Dufour eds. (2003). Assessing Microbial safety of drinking water: Improving approaches and methods. World Health Organization and organization for Economic Cooperation and Development, IWA Publishing.

[26]. Gerber. F (2010) An Economic Assessment of Drinking Water Safety Planning, Koror-Airai, Palau, SOPAC Technical Report 440, Published by Ocean \& Icelands Programme of SOPAC (Pacific Islands Applied Aeoscienc Commission), Fiji.

[27]. G.H. Huang, J. Xia (2001). Barriers to sustainable water-quality management, Journal of Environmental Engineering, Vol. 67, Issue 1, Jan. 2001, Page 1-23.

[28]. Goldriech EE (1996). Microbial quality of water supply in distribution systems. Boca Raton, FL, Lewis Publishers.

[29]. Goldriech EE, Lechevallier MW (1999). Microbial water quality in distribution systems, In: Letterman RD, ed. Water quality \& treatment $5^{\text {th }}$ ed. Newyork, McGraw-Hill.

[30]. Gregory R, Zabel TF, Edzwald JK (1999). Sedimentation and flotation, In: Letterman RD, ed. Water quality and treatment, Newyork, McGraw-Hill, Inc.

[31]. Gunnarsdottir M.J., Gararsson S, M., Elliot Sigmundsdottir, G, and Bartram J (2012), Benefits of water safety plans: Microbiology, Compliance and Public Health, Environmental Science \& Technology Vol 46.

[32]. Hall T, Watts M, Egerton A (2000). SPC and risk assessment techniques to optimize water treatment for cryptosporidium removal. Proceedings of the American water works Association water quality technology Conference.

[33]. Hambsch B, Werner P (1993). Control of bacterial regrowth in drinking water treatment plants and distribution system. Water supply.

[34]. Holf JC (1978). The relationship of turbidity to disinfection of potable water. Washington DC, United States Environmental Protection Agency.

[35]. IS 10500:2012 Indian Standard, Drinking Water-Speciation (Second Revision), Bureau of Indian Standards, New Delhi.

[36]. Kenneth T. Bogen and Robert C. Spear (1987), Integrating Uncertainty and Inter individual Variability in Environmental Risk Assessment, Risk Analysis, Vol. 7, Issue 4, (Dec. 1987), Pages 427-436.

[37]. Le Chevallier MW (1999). The case for maintaining a disinfectant residual. Journal of the American water works Association.

[38]. Le Chevallier MW, Evans TM, Seilder RJ (1981). Effect of turbidity on chlorination efficiency \& bacterial persistence in drinking water. Applied \& Environmental Microbiology.

[39]. Letterman RD, Amirtharajah A, O’Melia CR (1999). Coagulation and flocculation. N: Letterman RD, ed. Water quality and treatment, Newyork, McGraw Hill, Inc.

[40]. L. Failing, R. Gregory, M. Harstone (2007), Integrating science \& local knowledge in environmental risk management: A decisionfocused approach, Ecological Economics, Vol. 64, Issue 1, 15 Oct. 2007, Pages 47-60.

[41]. Logsdon GS (1990). Microbiology and water filtration, Newyork springer Vertag.

[42]. Logsdon GS, Hess AF, Chipps MJ (2000). Operating and monitoring pretreatment and filtration for optimized filter performance. Proceedings of the American water works Association water quality technology Conference.

[43]. Lubka Tchankova, (Faculty of Management, Technical University of Sofia, Bulgaria) (2002). Risk identification-basic stage in risk management, Environment Management \& Health, Vol. 13, Page 290-297.

[44]. Macharia. L. (2012), Ensuring water safety in informal settlements (Nairobi KENYA), IWA Water safety Conference, Nairobi.

[45]. Mark W Lechevallier and KWOK-Keing Au, WHO with IWA publishing, www.iwapublishing.com ISB M 9241562552 (WHO), Water treatment \& Pathogen Control-Process efficiency in achieving safe drinking water.

[46]. Mc Tigue et al. (1998). National Assessment of particle removal by filtration. Denver, CO, American water works Association Research Foundation and American water works Association.

[47]. Ministry of Health, Newzealand (2014), Small drinking-water supplies (Water safety kit) www.health.govt.nz.

[48]. Nabedaum DR, Chapman M, Merdan R, Rizak S. (2004), A guide to hazard identification \& risk assessment for drinking water supplies Research Report 11, CRC for water quality \& treatment. www.waterquality.crc.org.au/ publication occphr resrpts.htm.

[49]. Robbins RW et al. (1991). Effective water shed management for surface water supplies. Denver, CO, American water works Association Research Foundation and American water works Association.

[50]. S.J.T. Pollard, J.E. Strutt, B.H. Macgillivray, P.D. Hamilton, S.E. Hrudey (2004), Risk Analysis and Management in the Water Utility Sector: A Review of Drivers, Tools and Techniques, Process Safety and Environmental Protection, Vol. 82, Issue 6, Nov. 2004, Page 453-462.

[51]. Steve E. Hrudey \& William Leiss (2003). Risk Management \& Precaution: Insights on the Cautious Use of Evidence, Environ Health Perspect 111:1577-1581 (2003).

[52]. Susan R. Poulter (1998). Monte Carlo Simulation Environmental Risk Assessment - Science, Policy and Legal Issues. Risk: Health, Safety \& Environment.

[53]. Terrence Thompson et al. (2007), Chemical safety of drinking water, Assessing priorities or Risk Management.

[54]. Timothy O'Riordan, Steve Rayner (1991). Risk management for global environmental change.

[55]. USEPA (1991). Optimizing water treatment plant performance using the composite correction programme. Cincinnati, OH, office of Research and Development, United States Environmental Protection Agency.

[56]. Water \& Sanitation for Health Project (1998), Lessons learned from the WASH Project: ten years of water \& sanitation experience in developing countries (13 years) www.WASH,virginia.

[57]. Weber WJ Jr. (1972). Physiochemical process for water quality control. New York, John Wiley \& Sons.

[58]. WHO (2004) Guidelines for drinking-water quality $3^{\text {rd }}$ ed, World Health Organization, Geneva.

[59]. WHO (2006) Guidelines for drinking water quality $1^{\text {st }}$ Addendum to $3^{\text {rd }}$ ed, Vol. 1. Recommendations, World Health Organization. 
[60]. WHO WPR (2008) Training workbook on water safety plans for urban systems published by WHO Western Pacific Region. http://www.who.int/water sanitation health/(2011/dwq guidelines/en/

[61]. WHO (2011), Guidelines for drinking water quality; Fourth Edition http://www.who.int/water sanitation health/ (2011/dwq guidelines/en/

[62]. Wouter Poortinga, Anglia Linda Steg, Charles Vlek (2003), Environmental Risk Concern and Preferences for Energy-Saving Measures, Environ Health Perspect 111 (13):1577-1581 (Oct. 2003). 